\title{
Computer simulation of oscillatory processes of viscoelastic elements of thin-walled structures in a gas flow
}

\author{
Bakhtiyar Khudayarov ${ }^{1}$, Fozilzhon Turaev ${ }^{1}$, Olimzhon Kucharov ${ }^{2}$ \\ ${ }^{1}$ Department of Higher Mathematics, Tashkent institute of irrigation and agricultural mechanization \\ engineers, Tashkent, 100000, Uzbekistan \\ ${ }^{2}$ Scientific and Innovation Center of Information and Communication, Tashkent, 100000, Uzbekistan
}

\begin{abstract}
Results of numerical investigation of dynamic behavior of deformed wing aircraft in a gas flow are presented in the paper. Vibrations with respect to deflections are described by a system of integro-differential equations in partial derivatives. Using the Bubnov-Galerkin method, the problem is reduced to a system of ordinary integro-differential equations, where time is an independent variable. The solutions of integro-differential equations are determined by a numerical method based on the use of quadrature formulas. Computational algorithms and a package of applied programs have been created to solve problems on nonlinear flutter of viscoelastic elements of an aircraft. The reliability of the solution of the problem is confirmed by comparison with known numerical and analytical results. The effect of different boundary conditions on critical flutter velocity is studied. Critical velocity and critical flutter time of viscoelastic plates are determined. It is shown that the singularity parameter $\alpha$ affects not only the vibrations of viscoelastic systems, but also critical time and critical flutter velocity. It is stated that consideration of viscoelastic properties of plate material leads to $40-60 \%$ decrease in critical flutter velocity.
\end{abstract}

\section{Introduction}

Enormous scale of the development in aviation industry and shipbuilding necessitates the further development of the theory and practice of mathematical simulation. The study of structure material with viscoelastic and nonlinear properties, the consideration of which has great theoretical and practical importance, approximates the theory of calculation to the actual conditions of structure operation. Therefore, the problems of the theory of hereditary elasticity attract serious attention of researchers.

Of particular interest are the nonlinear problems of the theory of hereditary elasticity, which, apart from their practical importance, are of considerable scientific interest in the spheres of mechanics, mathematical physics and computational mathematics. This is due to the fact that nonlinear problems of the theory of hereditary elasticity are reduced to boundary and initial-boundary value problems for nonlinear weakly singular integral-

* Corresponding author: bakht-flpo@yandex.ru 
differential equations withpartial derivatives, the complete investigation of which is connected with the development of new methods for solving weakly singular integraldifferential equations.

The above-mentioned scientific problem gives grounds to assert that the development of adequate mathematical models, numerical methods and algorithms for solving nonlinear integral-differential equations of dynamic problems of the hereditary theory of viscoelasticity is actual.

In connection with this, the development of mathematical models of individual elements of aircraft made of composite material is becoming very important.

One of the main difficulties for a complete understanding of the supersonic flutter phenomenon is the fact that critical velocity of the flutter depends on a large number of parameters. At present, the difficulty in isolating many of these factors in experimental study does not allow us to obtain a satisfactory agreement between experimental and theoretical results.In literature there are numerous reviews of the problem under investigation. An extensive bibliography is given by Marco Amabili [1, 2], Farbod Alijani and Marco Amabili [3], Y. Wang and Z. M. Wang [4]. The development of problems on the flutter-plate strip, plates and panels with account of viscoelastic properties of structure material is reflected in publications by Mojtaba Asgari, Mohammad Reza Permoon, Hassan Haddadpou [5], Tytti Saksa et al. [6], Wei Tao Zhao, Tian JunYu, Xiao Dong Yang [7], Mouafo T.A. Robinson, Sarp Adali [8], Mouafo T.A. Robinson, Sarp Adali [9], Xiaochen Wang, Zhichun Yang, Wei Wang, WeiTian [10], B.Kh.Eshmatov et al. [11], M.Mirsaidov et al. [34, 35] and others. In [5-9], the Kelvin-Voigt model is used to describe the strain processes occurringin viscoelastic materials.

As is known, exact solutions to the problem of flutter, even in the simplest cases, are non-existent to this day. Therefore, there are different opinionsregarding the effect of viscoelastic properties of structure material on critical velocity of flutter [12- 15]. In [14, 15], theoretically (by average method), and in [12, 13] by computational experiment it is shown that the effect of viscoelastic properties of structure material on critical flutter velocity in both steady and transient processes leads to a significant decrease in value of critical velocity of a flutter.In $[16,17]$ solving the problem of the flutter of viscoelastic strip (in the case of the exponential kernel of heredity), the conclusion is drawn that critical flutter velocity, in both ideally elastic and viscoelastic cases, does not differ much, and the "viscous" properties of material affect the character of strip motion in subcritical regiononly. Similar conclusions have been established earlier in [12, 18, 19], and this phenomenon is explained by the fact that the exponential kernels do not correctly describe the hereditarily deformable properties of structure material.

This drawback lies in the fact that the relaxation kernel, proportional to the strain rate, has a finite value at initial time, however the experiment shows an arbitrarily high strain rate, i.e. at $t \rightarrow 0, R(t) \rightarrow \infty$, that contradicts the experiment [20-22], and as a result, when solving any dynamic problem (not only the flutter problem), errors accumulate over time and the results will be distorted in comparison with reality processes.In fact, in [23] it is exactly stated that the vibrations of any viscoelastic system (beams, plates and shells) under constant load occur along the creep curve and attenuate over time along this curve. This law is fulfilled if any weakly singular kernel of heredity of Abel-type is used as the kernel of heredity; the use of an exponential kernel over time due to errors accumulation leads to a severe violation of this law of motion of viscoelastic systems.

In $[12,18,19]$ it is shown that if to solve viscoelastic flutter problems with weakly singularkernels of heredity, then there immediately appears a significant effect of viscosity parameter and singularity on the values of critical velocity and critical time of both linear and nonlinear flutter problems. Therefore, the development of a new method for solving and demonstrating the reliability of results of the study of dynamic stability problem, both 
in ideally elastic and in hereditary deformable systems in a gas flow, is a very urgent problem. The present paper is devoted to the above problem. The accuracy and convergence of the method is tested on known results [24-26] related to the flutter of elastic plates and viscoelastic strips $[16,17]$.

\section{Formulation of the problem}

\subsection{Nonlinear flutter of viscoelastic plates}

Consider a rectangular viscoelastic plate with sides $a$ and $b$, which is flowed over from one side by a supersonic gas flow of velocity $V$. Aerodynamic pressure is taken into account by the piston theory of A.A. Ilyushin [27].

For the case of finite deflections of a plate commensurate with its thickness $h$, strains of viscoelastic plate are described by equations:

$$
\begin{gathered}
\frac{D}{h}\left(1-R^{*}\right) \nabla^{4} w=L(w, \Phi)-\rho \frac{\partial^{2} w}{\partial t^{2}}-\frac{B}{h} \frac{\partial w}{\partial t}-\frac{B V}{h} \frac{\partial w}{\partial x}-\frac{B_{1} V^{2}}{h}\left(\frac{\partial w}{\partial x}\right)^{2} \\
\frac{1}{E} \nabla^{4} \Phi=-\left(1-R^{*}\right) \frac{1}{2} L(w, w) .
\end{gathered}
$$

In accordance with the boundary conditions:

$G_{1}$ ) - hinged support on all edges

At $x=0, x=a$

$$
w=0, \quad \frac{\partial^{2} w}{\partial x^{2}}=0, \frac{\partial^{2} \Phi}{\partial y^{2}}=0, v=0,
$$

a t $y=0, y=b$

$$
w=0, \quad \frac{\partial^{2} w}{\partial y^{2}}=0, \frac{\partial^{2} \Phi}{\partial x^{2}}=0, u=0,
$$

$G_{2}$ ) - hinged support on two edges and fixing on the other two:

At $x=0, x=a$

$$
w=0, \quad \frac{\partial^{2} w}{\partial x^{2}}=0, \frac{\partial^{2} \Phi}{\partial y^{2}}=0, v=0,
$$

at $y=0, y=b$

$$
w=0, \quad \frac{\partial w}{\partial y}=0, u=0, \quad v=0,
$$

$G_{3}$ ) - rigid fixingon all edges:

At $x=0, x=a$

$$
w=0, \quad \frac{\partial w}{\partial x}=0, u=0, \quad v=0,
$$

at $y=0, y=b$

$$
w=0, \quad \frac{\partial w}{\partial y}=0, u=0, \quad v=0,
$$

solution of equation (1) is taken in the form: 


$$
\begin{aligned}
& w(x, y, t)=\sum_{n=1}^{N} \sum_{m=1}^{M} w_{n m} \varphi_{n m}(x, y), \\
& \Phi(x, y, t)=\sum_{n=1}^{N} \sum_{m=1}^{M} \Phi_{n m} \varphi_{n m}(x, y),
\end{aligned}
$$

Where $w_{n m}=w_{n m}(t)$ and $\Phi_{n m}=\Phi_{n m}(t)-$ are the sought for time functions; $\varphi_{n m}(x, y)-$ known functions, depending on boundary conditions:

$$
\begin{aligned}
& G_{1}: \quad \varphi_{n m}(x, y)=\sin \frac{n \pi x}{a} \sin \frac{m \pi y}{b} ; \\
& G_{2}: \quad \varphi_{n m}(x, y)=\sin \frac{n \pi \pi}{a}\left(1-\cos \frac{2 m \pi y}{b}\right) ; \\
& G_{3}: \quad \varphi_{n m}(x, y)=\left(\cos \frac{(n-1) \pi x}{a}-\cos \frac{(n+1) \pi x}{a}\right)\left(1-\cos \frac{2 m \pi \pi}{b}\right) .
\end{aligned}
$$

Integrating equation(1) by the Bubnov-Galerkin method with relationships (3) - (5), the following system of integro-differential equations (IDE) areobtainedwith respect to $w_{n m}(t)$ and $\Phi_{n m}(t)$ :

$$
\begin{gathered}
\sum_{n=1}^{N} \sum_{m=1}^{M} N_{k l n m}\left(\ddot{w}_{n m}+M_{\lambda} \dot{w}_{n m}\right)+\Omega\left(1-R^{*}\right) \sum_{n=1}^{N} \sum_{m=1}^{M} A_{k l n m} w_{n m}- \\
-\frac{M_{E}^{2} \lambda^{2}}{\lambda_{1}^{2}} \sum_{n, i=1}^{N} \sum_{m, r=1}^{M} B_{k l n m i r} w_{n m} \Phi_{i r}-M_{E}^{2} \beta_{1} \sum_{n=1}^{N} \sum_{m=1}^{M} D_{k l n m} \Phi_{n m}+ \\
+\aleph M^{*} M_{p}^{2} \lambda_{1} \sum_{n=1}^{N} \sum_{m=1}^{M} \gamma_{k l n m} w_{n m}+M_{1} M^{*^{2}} \sum_{n, i=1}^{N} \sum_{m, r=1}^{L} \Gamma_{k l n m i r} w_{n m} w_{i r}=0, \\
\sum_{n=1}^{N} \sum_{m=1}^{M} Q_{k \ln m} \Phi_{n m}= \\
=-\left(1-R^{*}\right)\left\{\sum_{n, i=1}^{N} \sum_{m, r=1}^{M} \lambda^{2} T_{k \ln m i r} w_{n m} w_{i r}+\lambda_{1}^{2} \beta_{1} \sum_{n=1}^{N} \sum_{m=1}^{M} S_{k \ln m} w_{n m}\right\},
\end{gathered}
$$

where

$$
\begin{gathered}
N_{k \ln m}=\int_{0}^{1} \int_{0}^{1} \phi_{n m} \phi_{k l} d x d y, \\
A_{k \ln m}=\int_{0}^{1} \int_{0}^{1}\left(\phi_{n m, x x x x}^{I V}+2 \lambda^{2} \phi_{n m, x x y y}^{I V}+\lambda^{4} \phi_{n m, y y y y}^{I V}\right) \phi_{k l} d x d y
\end{gathered}
$$




$$
\begin{aligned}
B_{k l n m i r}= & \int_{0}^{1} \int_{0}^{1}\left(\varphi_{n m, x x}^{\prime \prime} \psi^{\prime \prime}{ }_{i r, y y}+\varphi_{n m, y y}^{\prime \prime} \psi_{i r, x x}^{\prime \prime}-2 \varphi_{n m, x y}^{\prime \prime} \psi_{i r, x y}^{\prime \prime}\right) \varphi_{k l} d x d y, \\
D_{k l n m}= & \int_{0}^{1} \int_{0}^{1}\left(\lambda^{2} k_{x} \psi^{\prime \prime}{ }_{n m, y y}+k_{y} \psi_{n m, x x}^{\prime \prime}\right) \psi_{k l} d x d y, \quad \gamma_{k l n m}=\int_{0}^{1} \int_{0}^{1} \varphi_{n m}^{\prime} \varphi_{k l} d x d y, \\
\Gamma_{k \ln m i r} & =\int_{0}^{1} \int_{0}^{1} \phi_{n m, x}^{\prime} \phi_{i r, x}^{\prime} \phi_{k l} d x d y, \\
Q_{k \ln m} & =\int_{0}^{1} \int_{0}^{1}\left(\psi_{n m, x x x x}^{I V}+2 \lambda^{2} \psi_{n m, x x y y}^{I V}+\lambda^{4} \psi_{n m, y y y y}^{I V}\right) \psi_{k l} d x d y, \\
& T_{k \ln m i r}=\int_{0}^{1} \int_{0}^{1}\left(\phi_{n r, x x}^{\prime \prime} \phi_{i m, y y}^{\prime \prime}-\phi_{n m, x y}^{\prime \prime} \phi_{i r, x y}^{\prime \prime}\right) \psi_{k l} d x d y, \\
S_{k \ln m}= & \int_{0}^{1} \int_{0}^{1}\left(k_{x} \phi_{n m, y y}^{\prime \prime}+k_{y} \phi_{n m, x x}^{\prime \prime}\right) \psi_{k l} d x d y .
\end{aligned}
$$

Eliminating $\Phi_{n m}(t)$, from this system, the following nonlinear IDEisobtained with respect to thesoughtfor function $w_{n m}(t)$ :

$$
\begin{gathered}
\sum_{n=1}^{N} \sum_{m=1}^{M} N_{k \ln m}\left(\ddot{w}_{n m}+M_{\lambda} \dot{w}_{n m}\right)+ \\
+\Omega\left(1-R^{*}\right) \sum_{n=1}^{N} \sum_{m=1}^{M} A_{k \ln m} w_{n m}+ \\
+\frac{M_{E}^{2} \lambda^{4}}{\lambda_{1}^{2}} \sum_{n, i, j=1}^{N} \sum_{m, r, s=1}^{M} a_{k \ln m i r j s} w_{n m}\left(1-R^{*}\right) w_{i r} w_{j s}+ \\
+M_{E}^{2} \beta_{1} \lambda^{2} \sum_{n, i=1}^{N} \sum_{m, r=1}^{M} K_{k \ln m i r} w_{n m}\left(1-R^{*}\right) w_{i r}+ \\
+M_{E}^{2} \beta_{1} \lambda^{2} \sum_{n, i=1}^{N} \sum_{m, r=1}^{M} F_{k \ln m i r} w_{n m}\left(1-R^{*}\right) w_{i r}+ \\
+M_{E}^{2} \beta_{1}^{2} \lambda_{1}^{2} \sum_{n=1}^{N} \sum_{m=1}^{M} E_{k \ln m}\left(1-R^{*}\right) w_{n m}+ \\
+\aleph M^{*} M_{p}^{2} \lambda_{1} \sum_{n=1}^{N} \sum_{m=1}^{M} \gamma_{k l n m} w_{n m}+\aleph M_{1} M^{*^{2}} \sum_{n, i=1}^{N} \sum_{m, r=1}^{M} \Gamma_{k l n m i r} w_{n m} w_{i r}=0, \\
w_{n m}(\mathrm{O})=w_{o n m}, \quad \dot{w}(\mathrm{O})=\dot{w}_{o n m}, \quad n=\overline{1, N} ; m=\overline{\mathbf{1 , M}} .
\end{gathered}
$$

Here 


$$
\begin{aligned}
& a_{k \ln m i r j s}=P_{k \ln m} \cdot C_{k l i r j s} \cdot Q_{k \ln m}^{-1} ; \\
& K_{k \ln m i r}=D_{k \ln m} S_{k l i r} Q_{k \ln m}^{-1} ; \quad F_{k \ln m i r}=P_{k \ln m} S_{k l i r} Q_{k \ln m}^{-1} .
\end{aligned}
$$

Note that equations (7) are written in general form and are called the basic resolving IDE oftwo-dimensional problems of dynamics of viscoelastic systems. Many problems of vibrations and stability of viscoelastic plates arereduced to equations of the form (7).

Equations of motion (7) are integrated by a numerical method based on the use of quadrature formulas [18, 19, 23, 27-31]. Results of calculations are given in Tables 1 and 2 and are presented by the graphs in Figs. $1-4$.

For elastic plate flutter velocity is $990 \mathrm{~m} / \mathrm{s}\left(G_{1}\right), 1535 \mathrm{~m} / \mathrm{s}\left(G_{2}\right)$, and $1688 \mathrm{~m} / \mathrm{s}\left(G_{3}\right)$. These results practically coincide with the values obtained by analytical method in [23-25] $\left(G_{1}\right.$ : $\left.V_{\text {cr }}=969 \mathrm{~m} / \mathrm{s}, A_{1}=513 ; G_{2}: V_{\text {cr }}=1537 \mathrm{~m} / \mathrm{s} ; A_{1}=814 \mathrm{~m} / \mathrm{s} ; G_{3}: V_{\text {cr }}=1542 \mathrm{~m} / \mathrm{s} ; A_{1}=842\right)$. For viscoelastic plate with regular kernel of heredity, this velocity is $935 \mathrm{~m} / \mathrm{s}\left(G_{1}\right), 1442 \mathrm{~m} / \mathrm{s}$ $\left(G_{2}\right)$ and $1605 \mathrm{~m} / \mathrm{s}\left(G_{3}\right)$, respectively.

Comparison of different cases of plate fixation shows that with an increase in a number of fixed sides of the plate,flutter critical velocity increases.

It can be seen from the obtained results that if the exponential kernel $(\alpha=1)$ is used, flutter velocity decreases by approximately $5 \%$, and when the Koltunov-Rzhanitsyn kernel is used this velocity decreases by $45 \%$ relative to critical velocity of the flutter of ideally elastic plates. Therefore, when using exponential kernels, flutter velocity of viscoelastic plate practically coincides with critical flutter velocity for ideally elastic plates. These conclusions and results fully agreewith the conclusions and results given in $[16,17]$, where critical flutter velocities are determined by a numerical-analytical method.

Table 1. Effect of boundary conditions on flutter velocityof plate

\begin{tabular}{cccccc|c}
$\begin{array}{c}\text { Boundary } \\
\text { Conditions }\end{array}$ & $\boldsymbol{A}$ & $\boldsymbol{\alpha}$ & $\boldsymbol{\beta}$ & $\boldsymbol{t}_{\boldsymbol{c r}}$ & $\boldsymbol{V}_{\mathrm{cr}}(\boldsymbol{m} / \mathbf{s})$ \\
\hline \multirow{2}{*}{$\boldsymbol{G}_{\mathbf{1}}$} & 0 & - & - & 86 & 990 \\
& 0,01 & 1 & 0,1 & 107 & 935 \\
& 0,05 & 0,5 & 0,1 & 158 & 536 \\
\hline \multirow{3}{*}{$G_{2}$} & 0 & - & - & 98 & 1535 \\
& 0,01 & 1 & 0,1 & 123 & 1442 \\
& 0,05 & 0,5 & 0,1 & 185 & 871 \\
\hline \multirow{3}{*}{$G_{3}$} & 0 & - & - & 127 & 1688 \\
& 0,01 & 1 & 0,1 & 159 & 1605 \\
& 0,05 & 0,5 & 0,1 & 242 & 915 \\
\hline
\end{tabular}

According to the results obtained by many researchers [18, 19, 28, 29] using the integral stress-strain law with weakly singular kernelof heredity, it is obvious that the viscosity parameter leads to a decrease in critical velocity and an increase in critical time (Figs. 1,2). With vanishingly small internal friction, the velocity of the panel flutter is approximately 2 times less than the one calculated under the assumption that internal friction is completely absent $[12,13]$. The results obtained by the authors fully agreewith the conclusions and results given in $[13,16,17]$.

Table 2 shows the effect of the shape of plate deflection on critical velocity of the flutter. At $N=5$, critical velocity is $1540 \mathrm{~m} / \mathrm{s}$, and for $N=6$ this velocity is $1595 \mathrm{~m} / \mathrm{s}$. The difference is $3.5 \%$. 
Table 2. Effect of the shape of plate deflection on critical velocity of the flutter

\begin{tabular}{c|c|c|c|c|c|c}
\hline $\boldsymbol{A}$ & $\alpha$ & $\beta$ & $\lambda$ & $N$ & $\gamma$ & $V_{\text {cr }}$ \\
\hline & & & & 2 & & 835 \\
\hline \multirow{3}{*}{0,05} & 0,25 & 0,05 & 3 & 4 & 1 & 1130 \\
\hline & & & & 5 & & 1540 \\
\hline & & & 6 & & 1595 \\
\hline
\end{tabular}

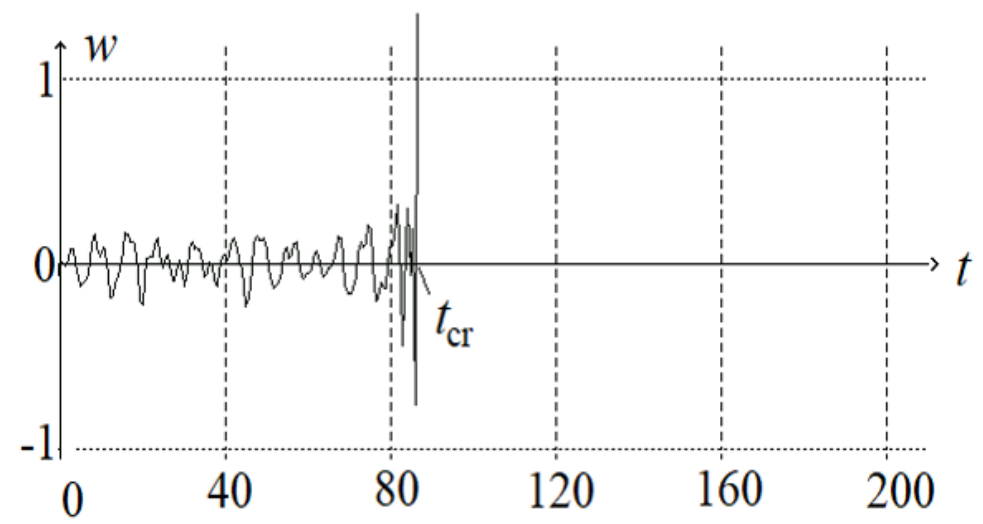

Fig. 1. Dependence of deflection on time at $A=0,0 ; V=V_{\text {cr. }}$.

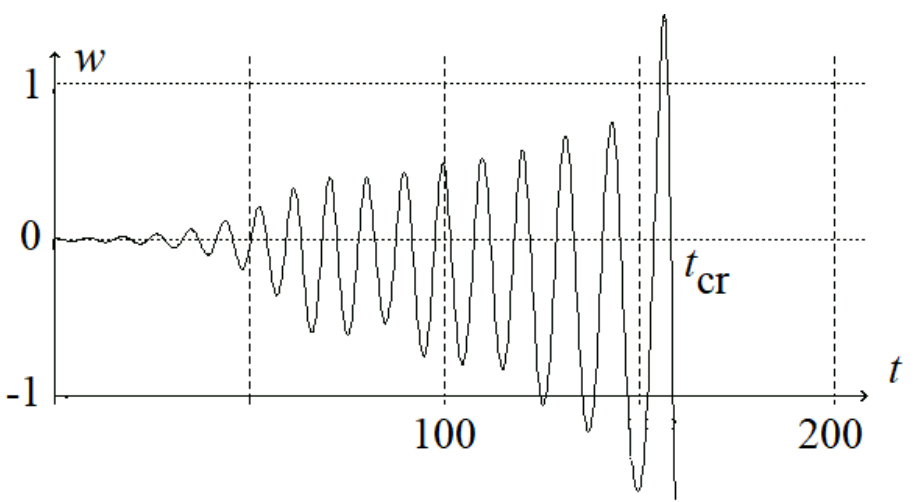

Fig. 2. Dependence of deflection on time at $A=0,05 ; \alpha=0,5 ; \beta=0,05 ; V=V_{\text {cr }}$

The study of viscosity effect is given. Calculations have shown that an account of viscous resistance leads to a decrease in critical value of the flutter. Fig.3 shows the effect of boundary conditions $G_{1}$ (curve 1), $G_{2}$ (curve 2), $G_{3}$ (curve 3 ) on vibrations at the midpoint of viscoelastic plate $(A=0.05)$ at $\lambda_{1}=180$. With an increase in a number of fixed sides of the plate at initial time, the amplitude of vibrations increases, but over time it attenuates earlier than in cases with hinged supports. 


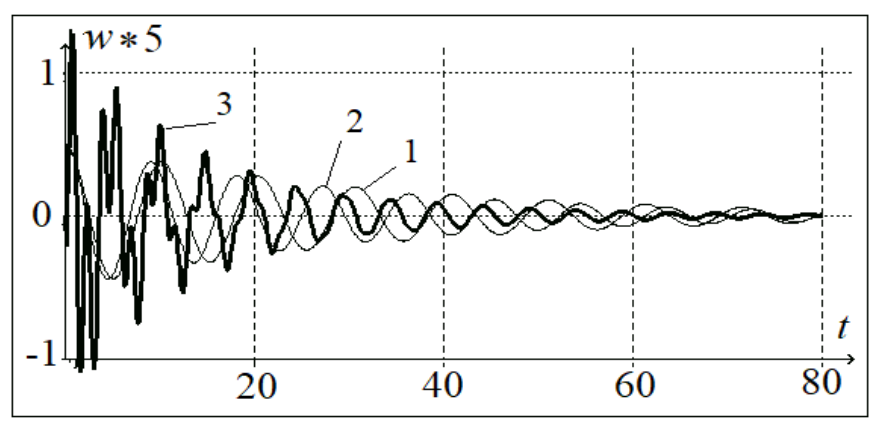

Fig. 3. Dependence of deflection on time at $G_{1}(1) ; G_{2}(2) ; G_{3}(3)$; $A=0,05 ; \alpha=0,25 ; \beta=0,05 ; \lambda_{1}=180 ; \lambda=1,2 ; V=560 \mathrm{~m} / \mathrm{s}$

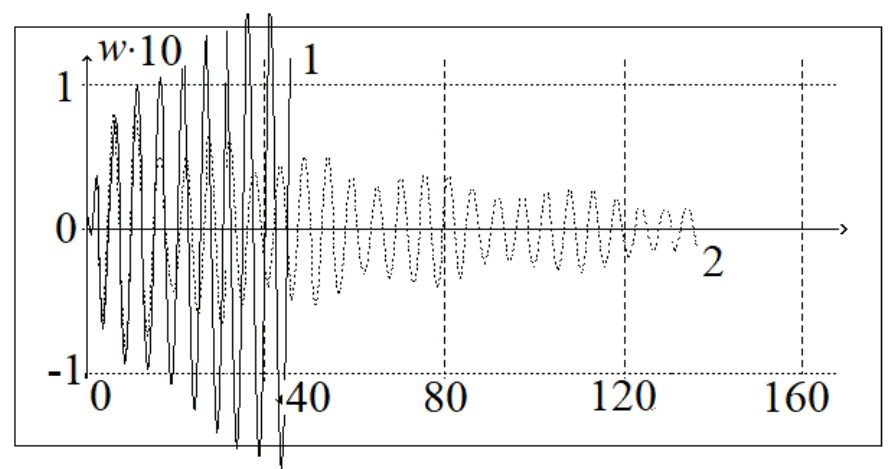

Fig. 4. Linear theory (1); nonlinear theory (2); $A=0.05 ; \alpha=0.25 ; \beta=0.05 ; \lambda=3 ; \lambda_{1}=300 ; V=1339 \mathrm{~m} / \mathrm{s}$

Fig. 4 shows the graphs of vibration amplitude as a function of time for studying the role of geometric and aerodynamic nonlinearity. In a linear statement, the amplitude of vibrations increases rapidly (curve 1), flow velocity is greater than critical one, and with account of nonlinearity, the amplitude of vibrations decreases (curve 2).

\subsection{The problem of vibrations and stability of viscoelastic strip}

Consider the problem of vibrations and stability of elastic and viscoelastic strip in a gas flow $[16,17]$ and present a comparative analysis of the results of solution with the ones obtained by the proposed method.

In a rectangular coordinate system, the strip occupies region $0 \leq y \leq l, x \geq 0$. On one side it is flown overby a gas flow with velocity vector $V=V \boldsymbol{n}_{\mathbf{0}}, \boldsymbol{n}_{\mathbf{0}}=\{\cos \theta, \sin \theta\}$.

Strip vibrations are described by equation $[16,17]$

$$
D\left(1-R^{*}\right) \nabla^{4} w+\rho h \frac{\partial^{2} w}{\partial t^{2}}+B \frac{\partial w}{\partial t}+B V \vec{n}_{0} \cdot \operatorname{grad} w=0 .
$$

Assuming that $R(t)=A \cdot \exp (-\beta t) \cdot t^{\alpha-1}$ and introducing the dimensionless coordinates $\frac{x}{l}, \frac{y}{l}$, time $\beta$ tand velocity $M^{*}=\frac{V}{V_{\infty}}$ given in $[16,17]$, and retaining the previous notations, equation (8) is written in the form: 


$$
\left(1-\lambda R_{1}^{*}\right) \nabla^{4} w+a_{3} M^{*} \vec{n}_{0} \cdot \operatorname{grad} w+a_{2} \frac{\partial w}{\partial t}+a_{1} \frac{\partial^{2} w}{\partial t^{2}}=0
$$

Here the notations $\lambda=\frac{A}{\beta}, \quad R_{1}=\exp (-t) \cdot t^{\alpha-1} \beta^{1-\alpha}, a_{1}=12\left(1-v^{2}\right) \frac{\beta^{2} l^{2} \rho}{h^{2} E}$ $, \quad a_{2}=12\left(1-v^{2}\right) \frac{\beta l^{4} p_{0}}{h^{3} V_{\infty} E}, \quad a_{3}=12\left(1-v^{2}\right) \frac{\gamma p_{0}}{E}\left(\frac{l}{h}\right)^{3}$ are introduced,and other notations correspond to the ones assumed in $[16,17]$.

Solution of equation (9) is chosen in the form:

$$
w=w(t) \cdot \exp \left(-\alpha_{1} x\right) \cdot \sin \pi y .
$$

Substituting (10) into (9) at $\theta=0$ we get

$$
\begin{gathered}
a_{1} \ddot{w}+a_{2} \dot{w}+\mu\left(1-\lambda R_{1}^{*}\right) w-\alpha_{1} a_{3} M w=0 \\
w(0)=C_{1}, \quad \dot{w}(0)=C_{2},
\end{gathered}
$$

where $\mu=\left(\alpha_{1}^{2}-\pi^{2}\right)^{2} ; C_{1}, C_{2}-$ are the known constants.

Equation (11) with initial conditions (12) is solved by a numerical method. This method is based on the use of various analytical transformations that allow initial systems to be reduced to the systems of integral equations with regular kernels and stable numerical integration ensuring the solution of problems with a high degree of accuracy.

According to numerical method, we regularize the IDE system (11) with singular kernels. Substituting the variables

$$
t-\tau=z^{\frac{1}{\alpha}}, 0 \leq z \leq t^{\alpha} \quad(0<\alpha<1)
$$

the integral with the Koltunov-Rzhanitsyn kernel with a singularity of the following form

$$
A \int_{0}^{t}(t-\tau)^{\alpha-1} \exp (-\beta(t-\tau)) w(\tau) d \tau
$$

has the form

$$
\frac{A}{\alpha} \int_{0}^{t^{\alpha}} \exp \left(-\beta z^{\frac{1}{\alpha}}\right) w\left(t-z^{\frac{1}{\alpha}}\right) d z
$$

Note that after substitution of variables, the integrand with respect to $z$ becomes regular. Then, assuming that $t=t_{i}, t_{i}=i \Delta t, i=1,2, \ldots(\Delta t=$ const -is the interpolation step $)$ and replacing the integrals by some quadrature formulas (in particular, of trapezoids), we get

$$
\frac{A}{\alpha} \sum_{k=0}^{i} B_{k} \exp \left(-\beta t_{k}\right) w_{i-k}
$$

where the coefficients are $B_{0}=\frac{\Delta t^{\alpha}}{2} ; \quad B_{i}=\frac{\Delta t^{\alpha}\left(i^{\alpha}-(i-1)^{\alpha}\right)}{2}$;

$$
B_{k}=\frac{\Delta t^{\alpha}\left((k+1)^{\alpha}-(k-1)^{\alpha}\right)}{2}, \quad k=\overline{1, i-1} .
$$

On the basis of this method, an algorithm for numerical solution of system (11) is described. Integrating system (11) twice with respect to $t$, it can be written in integral form; with rational transformation we eliminate the singular features of integral operator $R_{1}^{*}$. 
Then, assuming that $t=t_{i}, t_{i}=i \cdot \Delta \mathrm{t}, i=1,2, \ldots(\Delta \mathrm{t}=$ const $)$ and replacing the integrals with quadrature formulas of trapezoids for the calculation of $w_{i k}=w_{k}\left(t_{i}\right)$, the recurrence formulas for the Koltunov-Rzhanitsyn kernel are obtained:

$$
\begin{aligned}
& w_{p}=\frac{1}{1+A_{p}\left(a_{2} / a_{1}\right)}\left\{w_{0}+\left(\dot{w_{0}}+\left(a_{2} / a_{1}\right) w_{0}\right) t_{p}-\right. \\
& \left.-\frac{1}{a_{1}} \sum_{j=0}^{p-1} A_{j}\left(a_{2} w_{j}-\left(t_{p}-t_{j}\right)\left[\begin{array}{l}
\alpha_{1} a_{3} M w_{j}+ \\
+\mu\left(w_{j}-\lambda \sum_{s=0}^{j} B_{s} \exp \left(-t_{s}\right) \beta^{1-\alpha} w_{j-s}\right)
\end{array}\right]\right)\right\}
\end{aligned}
$$

With the proposed approach, in the algorithm for numerical solution of the problemthe factor $t_{p}-t_{j}$ at $j=p$ in formula (19), takes zero value, i.e. the last summand is zero. Therefore, the summation is done from zero top-1( $j=\overline{0, p-1}$ ).

Thus, according to numerical method with respect to the unknowns, a system of algebraic equations is obtained. The Gauss method is used to solve the system. Based on the developed algorithm, a package of applied computer programs is created. Results of calculations are given in Tables 3 .

Table 3. Dependence of critical velocity of strip flutter on the parameters of the kernel of heredity

\begin{tabular}{|c|c|c|c|}
\hline$\lambda$ & $\alpha$ & \multicolumn{3}{c}{$M_{c r}^{*}$} & $M_{c r}^{*}$ \\
\hline 0 & - & 0.096029 & 0.096027 \\
\hline 0.1 & 1 & 0.096065 & 0.096025 \\
\hline 0.1 & 0.1 & - & 0.093745 \\
\hline 0.1 & 0.03 & - & 0.0919745 \\
\hline 0.3 & 0.005 & - & 0.079220 \\
\hline
\end{tabular}

Table 3 shows the results of specific calculations for the parameters values $[16,17]$ :

$$
\frac{p_{0}}{E}=5 \cdot 10^{-7}, \rho=8 \cdot 10^{3} \mathrm{~kg} / \mathrm{m}^{3}, \gamma=1.4, v=0.3, V_{\infty}=330 \mathrm{~m} / \mathrm{s}, \frac{l}{h}=3 \cdot 10^{2} \text {. }
$$

The third column shows the results $[16,17]$ when critical flutter velocities are determined by a numerical-analytical method.

As seen from theresults obtained, for ideally elastic and viscoelastic strips (in the case of exponential kernel of heredity) the critical velocities of the flutter exactly coincide with the results given in $[16,17]$. For viscoelastic strip with a weakly singular heredity kernel, this velocity decreases.

\subsection{Results reliability of solving the dynamic stability problem of hereditary deformable systems}

Conclusions on results reliability of solving the dynamic stabilityproblem of hereditary deformable systems in a gas flow, obtained by eliminating weakly singular features of integral and integro-differential equations. 
Thus, the computational experiments carried out according to the algorithm of the proposed method for solving the flutter problem of viscoelastic systems completely refute some intuitive conclusions and natural dissatisfaction with the effect of viscoelastic properties on critical velocity and critical flutter time.Therefore, when solving dynamic problems of mechanics of a deformable rigid body, it is necessary to use the integral stress strain law with weakly singular heredity kernels of Abel-type. Numerical experiment of the dynamics of corresponding structures has shown the presence of a significant effect of this feature on the nature of their vibrations, for example, an account of weakly singular feature of heredity kernel leads to a significant decrease in the value of critical velocity and an increase in critical time (Figs. 5, 6). This fundamentally new mechanical effect may be of interest to the specialists in the field of designing such structures [33].

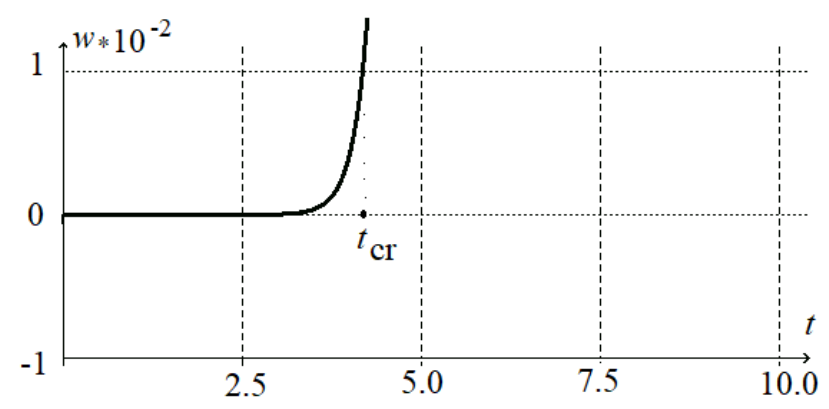

Fig. 5. Dependence of deflection on time at $\lambda=0,0 ; M_{c r}^{*}=0.096027$.

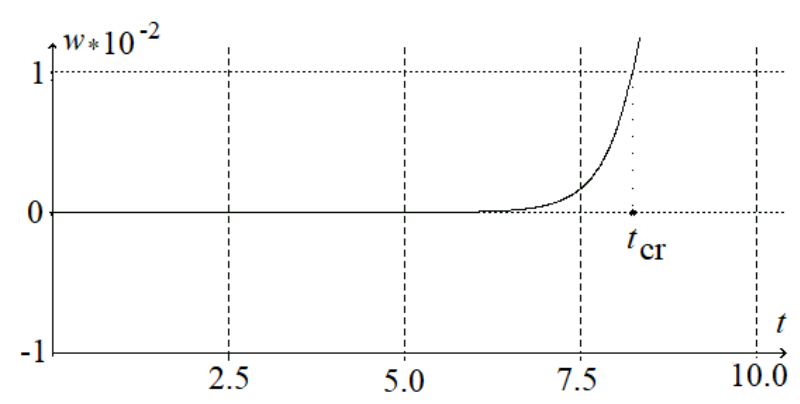

Fig. 6. Dependence of deflection on time at $\lambda=0,03 ; \alpha=0,005 ; M_{c r}^{*}=0.079220$.

\section{Conclusions}

It should be noted that the algorithm of the proposed method makes it possible to investigate in detail the effect of rheological parameters on the character of vibrational stability of hereditary deformed systems, in particular, in the study of flutter problem of ideally elastic systems.

As seen from Tables 1 and 3 the reliability of study results is proved by testing with known results related to the flutter of elastic plates [24, 26] and viscoelastic strip [16, 17]. In both cases, a satisfactory agreement of the solutions obtained is shown;that shows the reliability and high accuracy of the proposed calculation procedure. 


\section{References}

1. Marco Amabili, Nonlinear Vibrations and Stability of Shells and Plates. Cambridge University Press; 2008.

2. Marco Amabili,"Nonlinear Vibrations of Viscoelastic Rectangular Plates, "Journal of Sound and Vibration, vol.362, 2016, 142-156.

3. FarbodAlijani, Marco Amabili,"Non-linear Vibrations of Shells: A literature review from 2003 to 2013,"International Journal of Non-Linear Mechanics, vol.58, 2014, 233257.

4. Y.Wang, Z.M.Wang,"Stability of a Cracked Viscoelastic Plate of Varying Thickness Subjected to Follower Force, "Advanced Materials Research, 2011, 298-303.

5. Mojtaba Asgari, Mohammad Reza Permoon, Hassan Haddadpou,"Stability Analysis of a Fractional Viscoelastic Plate Strip in Supersonic Flow Under Axial Loading,"Meccanica, no.7, 2017.

6. Tytti Saksa, Juha Jeronen and Tero Tuovinen, "Stability of Moving Viscoelastic Panels Interacting with Surrounding Fluid,"Journal of Structural Mechanics, vol.45, no.3,2012, 88-103.

7. Wei Tao Zhao, Tian Jun Yu and Xiao Dong Yang,"Flutter Analysis of Viscoelastic Panels in Supersonic Flow,"Advanced Materials Research, vol.710, 2013,.256-259.

8. Mouafo Teifouet Armand Robinson, Sarp Adali,"Nonconservative Stability of Viscoelastic Plates Subject to Triangularly Distributed Follower Loads, "JOURNAL OF THEORETICAL AND APPLIED MECHANICS, vol.55, no.3,2017, 1015-1027. DOI: 10.15632/jtam-pl.55.3.1015

9. Mouafo Teifouet Armand Robinson, Sarp Adali,"Dynamic Stability of Viscoelastic Plates under Axial Flow by Differential Quadrature Method, "Engineering Computations,vol.34, no.4, 2017, 1240-1256. https://doi.org/10.1108/EC-03-20160113.

10. Xiaochen Wang, Zhichun Yang, Wei Wang, Wei Tian, "Nonlinear Viscoelastic Heated Panel Flutter with Aerodynamic Loading Exerted on Both Surfaces,"Journal Sound and Vibration, vol.409, no.24,2017, 306-317.https://doi.org/10.1016/j.jsv.2017.07.033

11. B.Kh.Eshmatov, Kh.Eshmatov, D.A.Khodzhaev,"Nonlinear flutter of viscoelastic rectangular plates and cylindrical panels of a composite with a concentrated masses,"Journal of Applied Mechanics and Technical Physics, vol.54, 2013, 578587. doi.org/10.1134/S0021894413040081

12. F.B.Badalov, Sh.F.Ganikhonov,"Vibrations of Hereditary-deformable Elements of Aircraft Designs, “ Tashkent; 2002.

13. G.G.Denisov, V.V.Novikov," On the Effect of Internal Friction on the Stability of Onedimensional Elastic Systems, in Dynamics of Systems, “ Publishing house of the Gorky University; 1975.

14. G.S.Larionov,"Nonlinear Flutter of Viscoelastic Plates,"Russ. Mech. Solids, no.4, 1975, 95-100.

15. V.I.Matyash,"Flutter of a Viscoelastic Plate,"Mech. Polym., no.6, 1971, 1077-1083.

16. I.A.Kiiko, V.V.Pokazeev,"Vibrations and Stability of Viscoelastic Strip Placed into Gas Flow,"Reports in Phys., vol.50, no.3, 2005, 158-160.

17.I.A.Kiiko, V.V. Pokazeyev, "Flutter of Viscoelastic Strip,"Journal of Engineering Mathematics, vol.78, no.1, 2013, 213-222. https://doi.org/10.1007/s10665-0129534-4

18. F.B.Badalov, A.Abdukarimov, B.A. Khudayarov,"Numerical Study of the Influence of Rheological Parameters on the Nature of Conventionally Deformed Systems,"Computational technologies, vol.12, no.4, 2007, 17-26. 
19.F.B.Badalov, B.A.Khudayarov, A.Abdukarimov, "Scientific Research and Development,"Problems of machine building and machine reliability, no.4, 2007, 107110.

20. M.A. Koltunov,“Creep and Relaxation,” Moscow: Higher School; 1976.

21. Yu.N.Rabotnov, "Elements of Hereditary Mechanics of Physical Bodies," Moscow: Science; 1977.

22. M.Yu.Ryazantseva,"On Wave Dispersion in an Infinite Elastic Three-layer Plate,"Proc. of the Russian Academy of Sciences. Mechanics of rigid body, no.1, 1998, 166-172.

23. F.B.Badalov,"Methods for Solving Integral and Integro-differential Equations of the Hereditary Theory of Viscoelasticity," Tashkent:Mekhnat; 1987.

24. A.A. Movchan, "On Oscillations of the Plate, Moving in a Gas, “J. Appl. Math. Mech., vol.20, 1956, 221-222.

25. A.A. Movchan,"Stability of a Blade Moving in a Gas,"Applied Mathematics and Mechanics, vol.21, no.5, 1957, 700-706.

26. K.K. Livanov, "Stability of Fixed Panel in a Supersonic Flow,"Engineering collection, vol.25, 1959, 101-103.

27. A.A. Il'yushin, "Flat Sections of the Law in High-speed Aerodynamics,"Applied mathematics and mechanics, vol.20, no.6, 1956, 733-755.

28. F.B.Badalov, B.A. Khudayarov,"Investigation of the Effect of Viscoelastic Properties of Material of an Aircraft Structures,"Proc. of NAS of Armenia. Series "Mechanics", vol.61, no.1, 2008, 75-82.

29. B.A. Khudayarov, "Numerical Analysis of Nonlinear Flutter of Viscoelastic Plates,"INTERNATIONAL APPLIED MECHANICS, vol.41, no.5, 2005, 538-542.

30. B.A. Khudayarov,N.G.Bandurin, "Numerical Investigation of Nonlinear Vibrations of Viscoelastic Plates and Cylindrical Panels in a Gas Flow, "Journal of Applied Mechanics and Technical Physics, vol.48, no.2, 2007, 279-284.

31. B.A. Khudayarov, "Flutter of Viscoelastic Plate in a Supersonic Gas Flow,"International Applied Mechanics, vol.46, no.4, 2010, 455-460.

32. F.B.Badalov, Kh.Eshmatov, M. Yusupov, "Some Methods of Solution the Systems of Integro-differential Equations in Problems of Viscoelasticity," Applied Mathematics and Mechanics, vol.51, no.5, 1987, 867-871.

33. Argicultural Aviation Research. NASA Conference Publication. 19-21 October 1976, Texas. 1977, 146.

34. M.M. Mirsaidov, T.Z. Sultanov, R.A. Abdikarimov, A.N. Ishmatov, B. Sh. Yuldoshev, E.S. Toshmatov, D.P. Jurayev, "Strength parameters of earth dams under various dynamic effects”, MAGAZINE OF CIVIL ENGINEERING, vol.77 no.1, 2018, 101-111.

35. M.M. Mirsaidov, T.Z. Sultanov, DF Rumi, "An assessment of dynamic behavior of the system" structure-foundation" with account of wave removal of energy", MAGAZINE OF CIVIL ENGINEERING, vol.39 no.4, 2013, 94-105. 